Cochrane Database of Systematic Reviews

\title{
Treatment for bleeding oesophageal varices in people with decompensated liver cirrhosis: a network meta-analysis (Protocol)
}

Roberts D, Tsochatzis E, Gurusamy KS

\section{Roberts D, Tsochatzis E, Gurusamy KS.}

Treatment for bleeding oesophageal varices in people with decompensated liver cirrhosis: a network meta-analysis. Cochrane Database of Systematic Reviews 2018, Issue 10. Art. No.: CD013155.

DOI: 10.1002/14651858.CD013155.

www.cochranelibrary.com 


\section{TABLE OF CONTENTS}

HEADER . . . . . . . . . . . . . . . . . . . . . . . . . . . . . . . . . . . . . . . . 1

ABSTRACT . . . . . . . . . . . . . . . . . . . . . . . . . . . . . . . . . . . . . . . 1

BACKGROUND . . . . . . . . . . . . . . . . . . . . . . . . . . . . . . . . . . 1

OBJECTIVES . . . . . . . . . . . . . . . . . . . . . . . . . . . . . . . . . . . . . .

METHODS . . . . . . . . . . . . . . . . . . . . . . . . . . . . . . . . . . . . . . .

ACKNOWLEDGEMENTS . . . . . . . . . . . . . . . . . . . . . . . . . . . . . . . . . . . . . . . .

REFERENCES . . . . . . . . . . . . . . . . . . . . . . . . . . . . . . . . . . . . . . 11

APPENDICES . . . . . . . . . . . . . . . . . . . . . . . . . . . . . . . . . . . . . . . . . . . . . . .

CONTRIBUTIONS OF AUTHORS . . . . . . . . . . . . . . . . . . . . . . . . . . . . . . . . . . . . . . . . . . . . 16

DECLARATIONS OF INTEREST . . . . . . . . . . . . . . . . . . . . . . . . . . . . . . . . . 17

SOURCES OF SUPPORT . . . . . . . . . . . . . . . . . . . . . . . . . . . . . . . . . . . . 17

NOTES . . . . . . . . . . . . . . . . . . . . . . . . . . . . . . . . . . . . . . . 17 
[Intervention Protocol]

\title{
Treatment for bleeding oesophageal varices in people with decompensated liver cirrhosis: a network meta-analysis
}

\author{
Danielle Roberts $^{1}$, Emmanuel Tsochatzis ${ }^{2}$, Kurinchi Selvan Gurusamy ${ }^{1}$ \\ ${ }^{1}$ Department of Surgery, Royal Free Campus, UCL Medical School, London, UK. ${ }^{2}$ Sheila Sherlock Liver Centre, Royal Free Hospital \\ and the UCL Institute of Liver and Digestive Health, London, UK
}

Contact address: Kurinchi Selvan Gurusamy, Department of Surgery, Royal Free Campus, UCL Medical School, Royal Free Hospital, Rowland Hill Street, London, NW3 2PF, UK. k.gurusamy@ucl.ac.uk.

Editorial group: Cochrane Hepato-Biliary Group.

Publication status and date: New, published in Issue 10, 2018.

Citation: Roberts D, Tsochatzis E, Gurusamy KS. Treatment for bleeding oesophageal varices in people with decompensated liver cirrhosis: a network meta-analysis. Cochrane Database of Systematic Reviews 2018, Issue 10. Art. No.: CD013155. DOI: 10.1002/14651858.CD013155.

Copyright (C) 2018 The Cochrane Collaboration. Published by John Wiley \& Sons, Ltd.

\begin{abstract}
A B S T R A C T
This is a protocol for a Cochrane Review (Intervention). The objectives are as follows:

To compare the benefits and harms of different treatments for bleeding oesophageal varices in people with decompensated liver cirrhosis.
\end{abstract}

\section{B A C K G R O U N D}

\section{Description of the condition}

\section{Liver cirrhosis}

The liver is a complex organ with multiple functions including carbohydrate metabolism, fat metabolism, protein metabolism, drug metabolism, synthetic functions, storage functions, digestive functions, excretory functions, and immunological functions (Read 1972). Liver cirrhosis is a liver disease in which the normal microcirculation, the gross vascular anatomy, and the hepatic architecture have been variably destroyed and altered with fibrous septa surrounding regenerated or regenerating parenchymal nodules (Tsochatzis 2014; NCBI 2018a). The major causes of liver cirrhosis include excessive alcohol consumption, viral hepatitis, non-alcohol-related fatty liver disease, autoimmune liver disease, and metabolic liver disease (Williams 2014; Ratib 2015; Setiawan 2016).

The global prevalence of liver cirrhosis is difficult to estimate as most estimates correspond to chronic liver disease (which includes liver fibrosis and liver cirrhosis). In studies from the US, the prevalence of chronic liver disease varies between $0.3 \%$ and 2.1\% (Scaglione 2015; Setiawan 2016); in the UK, the prevalence was $0.1 \%$ in one study (Fleming 2008). In 2010, liver cirrhosis was responsible for an estimated $2 \%$ of all global deaths, equivalent to one million deaths (Mokdad 2014). There is an increasing trend of cirrhosis-related deaths in some countries such as the UK, while there is a decreasing trend in other countries such as France (Mokdad 2014; Williams 2014). The major cause of complications and deaths in people with liver cirrhosis is due to the development of clinically significant portal hypertension (hepatic venous pressure gradient at least $10 \mathrm{mmHg}$ ) (de Franchis 2015). Some of the clinical features of decompensation include jaundice, coagulopathy, ascites, variceal bleeding, hepatic encephalopathy, and

Treatment for bleeding oesophageal varices in people with decompensated liver cirrhosis: a network meta-analysis (Protocol)

Copyright @ 2018 The Cochrane Collaboration. Published by John Wiley \& Sons, Ltd. 
renal failure (de Franchis 2015; McPherson 2016; EASL 2018). Decompensated cirrhosis is the most common indication for liver transplantation (Merion 2010; Adam 2012).

\section{Oesophageal varices}

Oesophageal varices are dilated blood vessels in the oesophagus, usually due to portal hypertension (NCBI 2018b), and are a feature of clinically significant portal hypertension. The prevalence of oesophageal varices varies between $40 \%$ and $95 \%$ in people with cirrhosis (Chawla 2012; McCarty 2017). The annual incidence of oesophageal varices in people with cirrhosis varies from $3 \%$ to 22\% (Cales 1990; Merli 2003; D’Amico 2014).

There are many classification systems available for assessing the risk of bleeding from oesophageal varices. The classification system that is followed from a management perspective is the Baveno I consensus definition which classifies oesophageal varices as small and large (de Franchis 1992). The criteria for distinction between small and large oesophageal varices is variable (de Franchis 1992). The current UK guidelines and European Association for the Study of the Liver (EASL) guidelines on the management of variceal bleeding acknowledges this variability and suggests that small varices tend to be narrow, and they flatten easily with air during endoscopy as compared to large varices which are usually broader and flatten with difficulty, or do not flatten at all (Tripathi 2015; EASL 2018). Other definitions for small oesophageal varices include less than 5 $\mathrm{mm}$ in size and less than $25 \%$ of oesophageal lumen (Abby Philips 2016). Other risk factors for bleeding from oesophageal varices include the pressure within the varices (hepatic venous pressure gradient at least $12 \mathrm{mmHg}$ ), increased tension on the variceal wall as indicated by red spots or red wale markings (longitudinal red streaks on the varices) on endoscopy, and severity of the liver disease (Beppu 1981; NIEC 1988; de Franchis 2015; Tripathi 2015). Approximately $15 \%$ to $20 \%$ of people with oesophageal varices bleed in about one to three years (Gluud 2012; Qi 2015). Shortterm mortality of an episode of acute variceal bleeding is about $15 \%$ to $30 \%$ (Ioannou 2003; Gøtzsche 2008; D’Amico 2010; Rios 2015). Five-year mortality in people with variceal bleeding in Taiwan was more than 80\% (Liu 2016). Mean in-hospital costs of treating acute episode of bleeding was EUR 13,500 in France in 2007 (Thabut 2007); mean six-month costs of treating people with variceal bleeding in USA was USD 16,500 in 2000 (Zaman 2000).

\section{Pathophysiology of oesophageal varices}

In addition to causing arterial vasodilation of the splanchnic circulation (dilation of the blood vessels supplying the digestive organs in the abdomen such as liver, pancreas, spleen, and intestines) (Gines 2009; Moore 2013), portal hypertension causes dilation of the collaterals between the portal venous system and systemic venous system (Sass 2009). One of the major locations of these collaterals is the lower end of the oesophagus and proximal part of the stomach. Therefore, portal hypertension leads to oesophageal varices (Sass 2009). According to the Frank's modification of the Laplace law, the tension on the walls of blood vessels is dependent upon the diameter of the blood vessel and the pressure gradient across the walls (that is, the difference in pressure inside the varices and the oesophageal pressure) (Herman 2015). Portal hypertension leads to an increase in both the diameter of the blood vessels and in the pressure at which blood flows in the varices; therefore, the tension on the walls of the blood vessels increases. This results in dilation of the blood vessels at the lower end of the oesophagus and proximal part of the stomach, which in turn increases the tension further (Herman 2015). This vicious circle can eventually culminate in rupture of the varices (Sass 2009; Herman 2015).

\section{Description of the intervention}

Treatments for acute oesophageal variceal bleeding can be broadly classified into four main categories: resuscitation and supportive interventions, pharmacological interventions, endoscopic interventions, and other interventions (de Franchis 2015; Tripathi 2015; Garcia-Tsao 2017; EASL 2018).

\section{Resuscitation and supportive interventions}

The initial management steps for a person experiencing acute variceal bleeding centre around resuscitation and stabilisation, usually in the intensive care setting (Ertel 2016). Decompensated liver disease complicates resuscitation because of the inability to tolerate volume shifts and susceptibility to dilutional coagulopathy (Ertel 2016). Therefore, there is some uncertainty regarding the optimum transfusion strategy approach and the target systolic blood pressure when administering intravenous fluids (Tripathi 2015). A restrictive transfusion strategy may be preferable to a liberal transfusion strategy (Villanueva 2013). Prophylactic broad-spectrum antibiotic therapy and prophylaxis for hepatic encephalopathy (for example, antibiotic and lactulose used concurrently), are also often used as supportive treatments to prevent complications in the management of people with bleeding varices (de Franchis 2015; Tripathi 2015; Garcia-Tsao 2017; EASL 2018).

\section{Pharmacological interventions}

The two major classes of drugs that have been used in the control of acute variceal bleeding include vasoconstrictors such as vasopressin or terlipressin; and somatostatin or its analogue octreotide (de Franchis 2015; Tripathi 2015; Garcia-Tsao 2017; EASL 2018). These are generally used in combination with endoscopic interventions (de Franchis 2015; Tripathi 2015; Garcia-Tsao 2017; EASL 2018). 


\section{Endoscopic interventions}

The two main endoscopic treatments for variceal haemorrhage are variceal band ligation and sclerotherapy (de Franchis 2015; Tripathi 2015; Garcia-Tsao 2017; EASL 2018).

\section{Other interventions}

Early transjugular intrahepatic portosystemic shunt (TIPS) should be considered for bleeding oesophageal varices at high risk of failure by pharmacological or endoscopic interventions (de Franchis 2015). Balloon tamponade and self-expanding oesophageal metal stents are generally reserved for refractory bleeding (bleeding not controlled by pharmacological or endoscopic interventions) (de Franchis 2015). Other treatments for refractory bleeding include surgical portosystemic shunts, surgical devascularisation procedures, and liver transplantation (Olson 2016).

\section{How the intervention might work}

Vasoactive medications act to decrease portal pressure by reducing portal blood flow (via splanchnic vasoconstriction) or reducing intrahepatic vascular resistance or both (Ioannou 2003; Tripathi 2015). The TIPS procedure and surgical portosystemic shunts are aimed at diverting blood flow from the portal system to the systemic circulation, thereby decreasing portal pressure and reducing oesophageal varices. Endoscopic interventions using variceal band ligation or sclerotherapy aim to achieve haemostasis by obliterating the varix and decreasing variceal wall tension (Ertel 2016). Balloon tamponade and removable oesophageal stenting methods apply direct pressure on the varices, and thus, mechanically stop the haemorrhage (Olson 2016).

\section{Why it is important to do this review}

Acute variceal haemorrhage is a medical emergency and can be life-threatening. The short-term mortality of an episode of acute variceal bleeding is about $15 \%$ to $30 \%$ (Ioannou 2003; Gøtzsche 2008; D'Amico 2010; Rios 2015). Furthermore, in the context of end-stage liver disease, a variceal bleed can precipitate other features of decompensation such spontaneous bacterial peritonitis, hepatic encephalopathy, and renal impairment, all of which can result in significant morbidity or mortality for patients (Perri 2016). There are several different treatment approaches available for treating bleeding oesophageal varices with significant uncertainty about their relative benefits and harms. Although there have been Cochrane Reviews focusing on the comparison of some of the treatments for bleeding oesophageal varices (Ioannou 2003; Khan 2006; Gøtzsche 2008; D'Amico 2010; Rios 2015), there have been no previous network meta-analyses on the topic. Network meta-analysis allows for a combination of direct and indirect evidence and the ranking of different interventions for different outcomes (Salanti 2011; Salanti 2012). With this systematic review and network meta-analysis, we aim to provide the best level of evidence for the benefits and harms of different treatments for acute bleeding oesophageal varices due to liver cirrhosis. If it is not possible to perform network meta-analysis in this review for any of the outcomes, we will use standard Cochrane methods to perform head-to-head comparison meta-analyses whenever possible. We will also present results from direct comparisons whenever possible, even if we perform the network meta-analysis.

\section{O B JE C T I VES}

To compare the benefits and harms of different treatments for bleeding oesophageal varices in people with decompensated liver cirrhosis.

\section{METHODS}

\section{Criteria for considering studies for this review}

\section{Types of studies}

We will consider only randomised clinical trials for this network meta-analysis irrespective of language, publication status, or date of publication. We will exclude studies of other design because of the risk of bias in such studies. Inclusion of indirect observational evidence could weaken our network meta-analysis, but this could also be viewed as a strength for assessing rare adverse events. It is well established that exclusion of non-randomised studies increases the focus on potential benefits and reduces the focus on the risks of serious adverse events and risks of any adverse events. However, because of the exponentially increased amount of work required for non-randomised studies, we will register and perform a new systematic review and meta-analysis of non-randomised studies for adverse events if there is uncertainty in the balance of benefits and harms of effective treatment(s).

\section{Types of participants}

We will include randomised clinical trials with adults with acutely bleeding oesophageal varices due to decompensated liver cirrhosis. We will include trials in which people with oesophageal varices also had gastric varices, but we will not include trials in which the treatment was targeted at the gastric varices rather than oesophageal varices. We will also exclude trials in which the participants had previously undergone liver transplantation. 


\section{Types of interventions}

We will include any of the following interventions for comparison with one another either alone or in combination.

- Vasopressin or analogues, that is, terlipressin (systemic vasoconstrictors).

- Somatostatin or analogues, that is, octreotide, vapreotide, lanreotide (systemic vasoconstrictors).

- Endoscopic variceal band ligation (obliterate varix mechanically).

- Endoscopic sclerotherapy (obliterate varix chemically by inducing inflammation or by using a glue).

- Endoscopic spray treatment using haemostatic powder (tissue adhesive that promotes clotting).

- Balloon tamponade (mechanical haemostasis).

- Removable self-expanding oesophageal stents (mechanical haemostasis).

- TIPS procedure (decrease portal hypertension).

- Surgical shunt creation or devascularisation procedures (decrease portal hypertension).

- Surgical devascularisation procedures (decrease portal hypertension).

- Tranexamic acid (antifibrinolytic).

- No active intervention (no intervention or use of placebo or only supportive treatment).

The above list is not exhaustive. If we identify treatments of which we were unaware, we will consider eligibility of the treatments for inclusion in the network if they are used primarily for treatment of acute variceal bleeding. We will report the findings for these interventions in the 'Results' and 'Discussion' sections of the review. We will exclude supportive treatments and interventions aimed at secondary prophylaxis from this review.

We will evaluate the plausibility of transitivity assumption by looking at the inclusion and exclusion criteria in the studies. Transitivity assumption is the assumption that participants included in the different trials with different treatments for bleeding oesophageal varices can be considered to be a part of a multi-arm randomised clinical trial and could potentially have been randomised to any of the interventions (Salanti 2012). In other words, any participant that meets the inclusion criteria is, in principle, equally likely to be randomised to any of the above eligible interventions. This necessitates that information on potential effect-modifiers such as previous history of oesophageal bleeding, interval from the onset of bleeding, presence or absence of other features of decompensation such as ascites, and the cointerventions (that is, use of prophylactic antibiotics) are the same across trials.

\section{Types of outcome measures}

\section{Primary outcomes}

- Proportion of participants who died due to any cause (allcause mortality).

- Health-related quality of life as defined in the included trials using a validated scale such as the European Quality of Life - 5 Dimentions (EQ-5D) or 36-Item Short Form Health Survey (SF-36) (EuroQol 2018; Optum 2018).

- Serious adverse events. We define a serious adverse event as any event that would increase mortality; is life-threatening; requires hospitalisation; results in persistent or significant disability; is a congenital anomaly/birth defect; or any important medical event that might jeopardise the person or require intervention to prevent it (ICH-GCP 1997). However, we will use the definitions used by study authors for serious adverse events.

- Proportion of people with one or more serious adverse events.

- Number of serious adverse events per participant.

\section{Secondary outcomes}

- Any adverse events: we define an adverse event as any untoward medical occurrence not necessarily having a causal relationship with the intervention but resulting in a dose reduction or discontinuation of intervention (any time after commencement of intervention) (ICH-GCP 1997). However, we will use the definition used by study authors for adverse events.

- Proportion of people with one or more adverse events.

- Number of any adverse events per participant.

- Proportion of participants with variceal rebleeding at six weeks (however defined by study authors).

- Symptomatic variceal bleeding (for example, shortness of breath, shock).

- Any variceal bleeding.

- Proportion of participants with other features of decompensation at six weeks.

\section{Exploratory outcomes}

- Requirement for additional treatments to control the acute bleeding episode.

- Blood transfusion requirements (whole blood or red cell concentrate - all episodes of bleeding within six weeks).

- Proportion of participants requiring blood transfusion.

- Amount of blood transfused.

- Length of hospital stay (all hospital admissions).

- Number of days of lost work (in people who work).

- Treatment costs (including the cost of the treatment and any resulting complications).

All primary, secondary, and exploratory outcomes will be assessed until 90 days.

Treatment for bleeding oesophageal varices in people with decompensated liver cirrhosis: a network meta-analysis (Protocol) 
We have chosen outcomes based on their importance to patients in a survey related to research priorities for people with liver diseases (Gurusamy 2018), based on feedback of the patient and public representative of this project, and based on an online survey about the outcomes promoted through Cochrane Consumer Network.

\section{Search methods for identification of studies}

\section{Electronic searches}

We will search the Cochrane Central Register of Controlled Trials (CENTRAL) in the Cochrane Library, MEDLINE Ovid, Embase Ovid, and Science Citation Index Expanded (Web of Science) from inception to date of search for randomised clinical trials comparing two or more of the above interventions without applying any language restrictions (Royle 2003). We will search for all possible comparisons formed by the interventions of interest. To identify further ongoing or completed trials, we will also search clinicaltrials.gov, and the World Health Organization International Clinical Trials Registry Platform (apps.who.int/ trialsearch/), which searches various trial registers, including ISRCTN and ClinicalTrials.gov. We will also search the European Medical Agency ( EMA) ( www.ema.europa.eu/ema/) and US Food and Drug Administration ( FDA) (www.fda.gov) registries for randomised clinical trials. The provisional search strategies are provided in Appendix 1.

\section{Searching other resources}

We will search the references of the identified trials and the existing Cochrane Reviews on bleeding oesophageal varices to identify additional trials for inclusion.

\section{Data collection and analysis}

\section{Selection of studies}

Two review authors (KG and DR) will independently identify trials for inclusion by screening the titles and abstracts and seek full-text articles for any references identified by at least one of the review authors for potential inclusion. We will select trials for inclusion based on the full-text articles. We will provide the list of references that we exclude and the reasons for their exclusion in the 'Characteristics of excluded studies' table. We will also list any ongoing trials identified primarily through the search of the clinical trial registers for further follow-up. We will resolve any discrepancies through discussion.

\section{Data extraction and management}

Two review authors (KG and DR) will independently extract the data below in a prepiloted Microsoft Excel-based data extraction form (after translation of non-English articles).

- Outcome data (for each outcome and for each intervention group whenever applicable):

- number of participants randomised;

- number of participants included for the analysis;

$\circ$ number of participants with events for binary

outcomes, mean and standard deviation for continuous outcomes, number of events for count outcomes;

$\circ$ definition of outcomes or scale used if appropriate.

- Data on potential effect modifiers:

$\circ$ participant characteristics such as age, sex, previous history of bleeding oesophageal varices, presence of other features of decompensation such as ascites, the aetiology for cirrhosis, and the interval between diagnosis of variceal bleeding and treatment;

o details of the intervention and control (including dose, frequency, and duration);

○ length of follow-up;

- information related to risk of bias assessment (see

Assessment of risk of bias in included studies).

- Other data:
$\circ$ year and language of publication;
o country in which the participants were recruited;
- year(s) in which the trial was conducted;
$\circ$ inclusion and exclusion criteria.

We will collect outcomes at maximum follow-up but also at shortterm (up to three months) and medium-term (from three months to five years) if this is available.

We will contact the trial authors in the case of unclear or missing information. If there is any doubt as to whether trials shared the same participants, completely or partially (by identifying common authors and centres), we will attempt to contact the trial authors to clarify whether the trial report was duplicated. We will resolve any differences in opinion through discussion.

\section{Assessment of risk of bias in included studies}

We will follow the guidance in the Cochrane Handbook for Systematic Reviews of Interventions (Higgins 2011) and described in the Cochrane Hepato-Biliary Group Module (Gluud 2018) to assess the risk of bias in included trials. Specifically, we will assess sources of bias as defined below (Schulz 1995; Moher 1998; Kjaergard 2001; Wood 2008; Savović 2012a; Savović 2012b; Lundh 2017; Savović 2018).

\section{Allocation sequence generation}

- Low risk of bias: the study authors performed sequence generation using computer random number generation or a random number table. Drawing lots, tossing a coin, shuffling 
cards, and throwing dice will be adequate if performed by an independent person not otherwise involved in the study. In general, we will classify the risk of bias as low if the method used for allocation concealment suggested that it was extremely likely that the sequence was generated randomly (for example, use of interactive voice response system).

- Unclear risk of bias: the study authors did not specify the method of sequence generation.

- High risk of bias: the sequence generation method was not random.

\section{Allocation concealment}

- Low risk of bias: the participant allocations could not have been foreseen in advance of, or during, enrolment. A central and independent randomisation unit controlled allocation. The investigators were unaware of the allocation sequence (for example, if the allocation sequence was hidden in sequentially numbered, opaque, and sealed envelopes).

- Unclear risk of bias: the study authors did not describe the method used to conceal the allocation so that the intervention allocations may have been foreseen before, or during, enrolment.

- High risk of bias: it is likely that the investigators who assigned the participants knew the allocation sequence. We will exclude such quasi-randomised studies.

\section{Blinding of participants and personnel}

- Low risk of bias: blinding of participants and key study personnel ensured, and it was unlikely that the blinding could have been broken; or rarely no blinding or incomplete blinding, but the review authors judged that the outcome was not likely to be influenced by lack of blinding.

- Unclear risk of bias: any of the following: insufficient information to permit judgement of 'low risk' or 'high risk;' or the trial did not address this outcome.

- High risk of bias: any of the following: no blinding or incomplete blinding, and the outcome was likely to have been influenced by lack of blinding; or blinding of key study participants and personnel attempted, but likely that the blinding could have been broken, and the outcome was likely to be influenced by lack of blinding.

\section{Blinded outcome assessment}

- Low risk of bias: blinding of outcome assessment ensured, and unlikely that the blinding could have been broken; or rarely no blinding of outcome assessment, but the review authors judged that the outcome measurement was not likely to be influenced by lack of blinding.

- Unclear risk of bias: any of the following: insufficient information to permit judgement of 'low risk' or 'high risk;' or the trial did not address this outcome.
- High risk of bias: any of the following: no blinding of outcome assessment, and the outcome measurement was likely to be influenced by lack of blinding; or blinding of outcome assessment, but likely that the blinding could have been broken, and the outcome measurement was likely to be influenced by lack of blinding.

\section{Incomplete outcome data}

- Low risk of bias: missing data were unlikely to make treatment effects depart from plausible values. The study used sufficient methods, such as multiple imputation, to handle missing data.

- Unclear risk of bias: there was insufficient information to assess whether missing data in combination with the method used to handle missing data were likely to induce bias on the results.

- High risk of bias: the results were likely to be biased due to missing data.

\section{Selective outcome reporting}

- Low risk of bias: the trial reported the following predefined outcomes: at least one of the outcomes related to the main reason for treatment of people with acute variceal bleeding, namely, allcause mortality or prevention of variceal rebleeding along with adverse events. If the original trial protocol was available, the outcomes should have been those called for in that protocol. If the trial protocol was obtained from a trial registry (for example, ClinicalTrials.gov), the outcomes sought should have been those enumerated in the original protocol if the trial protocol was registered before or at the time that the trial was begun. If the trial protocol was registered after the trial was begun, those outcomes will not be considered to be reliable.

- Unclear risk of bias: not all predefined, or clinically relevant and reasonably expected, outcomes were reported fully, or it was unclear whether data on these outcomes were recorded or not.

- High risk of bias: one or more predefined or clinically relevant and reasonably expected outcomes were not reported, despite the fact that data on these outcomes should have been available and even recorded.

\section{For-profit bias}

- Low risk of bias: the trial appeared free of industry sponsorship or other type of for-profit support that could manipulate the trial design, conductance, or results of the trial (industry-sponsored trials overestimate the efficacy by about 25\%) (Lundh 2017).

- Unclear risk of bias: the trial may or may not have been free of for-profit bias, as no information on clinical trial support or sponsorship was provided. 
- High risk of bias: the trial was sponsored by industry or received other type of for-profit support.

\section{Other bias}

- Low risk of bias: the trial appeared free of other components that could have put it at risk of bias (for example, inappropriate control or dose or administration of control, baseline differences, early stopping).

- Unclear risk of bias: the trial may or may not have been free of other components that could have put it at risk of bias.

- High risk of bias: there were other factors in the trial that could have put it at risk of bias (for example, baseline differences, early stopping).

We will consider a trial at low risk of bias if we assess the trial at low risk of bias across all listed bias risk domains. Otherwise, we will consider trials to be at high risk of bias. At the outcome level, we will classify an outcome at low risk of bias if the allocation sequence generation; allocation concealment; blinding of participants, healthcare professionals, and outcome assessors; incomplete outcome data; and selective outcome reporting (at the outcome level) are at low risk of bias for objective and subjective outcomes (Savović́ 2018).

\section{Measures of treatment effect}

\section{Relative treatment effects}

For dichotomous variables (for example, proportion of participants with serious adverse events or any adverse events), we will calculate the odds ratio (OR) with $95 \%$ credible interval (CrI) (or Bayesian confidence interval) (Severini 1993). For continuous variables (for example, health-related quality of life reported on the same scale), we will calculate the mean difference (MD) with 95\% Crl. We will use standardised mean difference (SMD) values with $95 \% \mathrm{Crl}$ for health-related quality of life if included trials have used different scales. For count outcomes (for example, number of serious adverse events or number of any adverse events), we will calculate the rate ratio (RaR) with 95\% Crl.

\section{Relative ranking}

We will estimate the ranking probabilities for all interventions of being at each possible rank for each intervention. We will obtain the surface under the cumulative ranking curve (SUCRA) (cumulative probability), rankogram, and relative ranking table with 95\% CrI for the ranking probabilities (Salanti 2011; Chaimani 2013).

\section{Unit of analysis issues}

The unit of analysis will be the participant undergoing treatment for bleeding oesophageal varices according to the intervention group to which the participant is randomly assigned.

\section{Cluster randomised clinical trials}

We will include cluster randomised clinical trials provided that the effect estimate adjusted for cluster correlation is available. If this is not available, we will include such trials if sufficient information to calculate the design effect is available from the trial because this will allow us to take clustering into account. We will also assess additional domains of risk of bias for cluster randomised trials according to guidance in the Cochrane Handbook for Systematic Reviews of Interventions (Higgins 2011).

\section{Cross-over randomised clinical trials}

If we identify any cross-over randomised clinical trials, we will include the outcomes after the period of first intervention because the included treatments can have residual effects.

\section{Trials with multiple intervention groups}

We will collect data for all trial intervention groups that meet the inclusion criteria. The codes we will use for analysis will account for the correlation between the effect sizes from studies with more than two groups.

\section{Dealing with missing data}

We will perform an intention-to-treat analysis whenever possible (Newell 1992); otherwise, we will use the data available to us. This may result in the use of 'per-protocol' analyses. Since these may be biased, particularly if the data are not missing at random (for example, treatment was withdrawn due to adverse events or duration of treatment was shortened because of lack of response and such participants were excluded from analysis), we will conduct bestworst case scenario analysis (good outcome in intervention group and bad outcome in control group) and worst-best case scenario analysis (bad outcome in intervention group and good outcome in control group) as sensitivity analyses whenever possible for dichotomous outcomes.

For continuous outcomes, we will impute the standard deviation from $\mathrm{P}$ values according to guidance in the Cochrane Handbook for Systematic Reviews of Interventions (Higgins 2011). If the data are likely to be normally distributed, we will use the median for meta-analysis when the mean is not available. If it is not possible to calculate the standard deviation from the P value or the confidence intervals, we will impute the standard deviation using the largest standard deviation in other trials for that outcome. This form of imputation can decrease the weight of the study for calculation of 
MDs and may bias the effect estimate to no effect for calculation of SMDs (Higgins 2011).

\section{Assessment of heterogeneity}

We will assess clinical and methodological heterogeneity by carefully examining the characteristics and design of included trials. We will assess the presence of clinical heterogeneity by comparing effect estimates (see Subgroup analysis and investigation of heterogeneity) in trial reports of different drug dosages, previous history of bleeding, interval between onset of bleeding and treatment, presence of other features of decompensation (for example, ascites), different aetiologies for cirrhosis (for example, alcohol-related liver disease, viral liver diseases, autoimmune liver disease), and based on the cointerventions (for example, both groups receive prophylactic antibiotics to decrease the risk of subacute bacterial peritonitis in people with low-protein ascites). Different study designs and risk of bias can contribute to methodological heterogeneity.

We will assess statistical heterogeneity by comparing the results of the fixed-effect model meta-analysis and the random-effects model meta-analysis, between-study standard deviation ( $\mathrm{tau}^{2}$, and comparing this with values reported in study of the distribution of between-study heterogeneity) (Turner 2012), and by calculating the $\mathrm{I}^{2}$ statistic using Stata/SE 14.2. If we identify substantial clinical, methodological, or statistical heterogeneity, we will explore and address the heterogeneity in subgroup analysis (see Subgroup analysis and investigation of heterogeneity).

\section{Assessment of transitivity across treatment comparisons}

We will assess the transitivity assumption by comparing the distribution of the potential effect modifiers (clinical: previous history of bleeding, interval between onset of bleeding and treatment, presence of other features of decompensation (for example, ascites); methodological: risk of bias, year of randomisation, duration of follow-up) across the different pair-wise comparisons.

\section{Assessment of reporting biases}

For the network meta-analysis, we will perform a comparisonadjusted funnel plot. If there is no meaningful way in which to rank these studies (that is, there was no specific change in the risk of bias in the studies, sample size, or the control group used over time), we will judge the reporting bias by the completeness of the search (Chaimani 2012).

\section{Data synthesis}

\section{Methods for indirect and mixed comparisons}

We will conduct network meta-analyses to compare multiple interventions simultaneously for each of the primary and secondary outcomes. Network meta-analysis combines direct evidence within trials and indirect evidence across trials (Mills 2012). We will obtain a network plot to ensure that the trials are connected by interventions using Stata/SE 14.2 (Chaimani 2013). We will exclude any trials that are not connected to the network from the network meta-analysis and report only the direct pair-wise meta-analysis for such comparisons. We will summarise the population and methodological characteristics of the trials included in the network meta-analysis in a table based on pair-wise comparisons. We will conduct a Bayesian network meta-analysis using the Markov chain Monte Carlo method in OpenBUGS 3.2.3 as per guidance from the National Institute for Health and Care Excellence (NICE) Decision Support Unit (DSU) documents (Dias 2016). We will model the treatment contrast (that is, log OR for binary outcomes, MD or SMD for continuous outcomes, log RaR for count outcomes, and log hazard ratio for time-to-event outcomes) for any two interventions ('functional parameters') as a function of comparisons between each individual intervention and the reference group ('basic parameters') using appropriate likelihood functions and links (Lu 2006). We will use binomial likelihood and logit links for binary outcomes, Poisson likelihood and log links for count outcomes, binomial likelihood and complementary log-log links (a semiparametric model which excludes censored participants from the denominator of 'at risk' participants at the point when they are censored), and normal likelihood and identity links for continuous outcomes. We will use the 'vasoactive drugs plus endoscopic ligation' as the reference group. We will perform a fixed-effect model and random-effects model for the network meta-analysis. We will report both models for comparison with the reference group in a forest plot. For each pairwise comparison in a table, we will report the fixed-effect model if the two models report similar results; otherwise, we will report the more conservative model.

We will use a hierarchical Bayesian model using three different initial values, employing codes provided by NICE DSU (Dias 2016). We will use a normal distribution with large variance $(10,000)$ for treatment effect priors (vague or flat priors). For the randomeffects model, we will use a prior distributed uniformly (limits: zero to five) for between-trial standard deviations but will assume same between-trial standard deviations across treatment comparisons (Dias 2016). We will use a 'burn-in' of 10,000 simulations, check for convergence (of effect estimates and between-study heterogeneity) visually (that is, whether the values in different chains mix very well by visualisation), and run the models for another 10,000 simulations to obtain effect estimates. If we do not obtain convergence, we will increase the number of simulations for the 'burn-in.' If we still do not obtain convergence, we will use alternate initial values and priors employing methods suggested by van Valkenhoef 2012. We will estimate the probability that each intervention ranks at one of the possible positions using the NICE 
DSU codes (Dias 2016).

\section{Assessment of inconsistency}

We will assess inconsistency (statistical evidence of the violation of transitivity assumption) by fitting both an inconsistency model and a consistency model. We will use inconsistency models employed in the NICE DSU manual as we will use a common between-study standard deviation (Dias 2014). In addition, we will use design-by-treatment full interaction model and inconsistency factor (IF) plots to assess inconsistency (Higgins 2012; Chaimani 2013). We will use Stata/SE 14.2 to create IF plots. In the presence of inconsistency, we will assess whether the inconsistency was due to clinical or methodological heterogeneity by performing separate analyses for each of the different subgroups mentioned in the Subgroup analysis and investigation of heterogeneity section.

If there is evidence of inconsistency, we will identify areas in the network where substantial inconsistency might be present in terms of clinical and methodological diversities between trials and, when appropriate, limit network meta-analysis to a more compatible subset of trials.

\section{Direct comparison}

We will perform the direct comparisons using the same codes and the same technical details.

\section{Calculation of required information size and Trial Sequential Analysis}

For calculation of the required information size, see Appendix 2. We will perform Trial Sequential Analysis for direct comparisons to control the risk of random errors when at least two trials are included for the comparison of other interventions versus 'vasoactive drugs plus endoscopic ligation' ('control') for the outcomes allcause mortality at maximal follow-up and health-related quality of life, the two outcomes that determine whether the intervention should be given (Wetterslev 2008; Thorlund 2011; TSA 2011; Wetterslev 2017). For all-cause mortality at maximal follow-up, we will use an alpha error as per guidance of Jakobsen 2014 (that is, 0.033 ), power of $90 \%$ (beta error of 10\%) (Castellini 2017), a relative risk reduction of $20 \%$, the median control group proportion observed in the trials, and the heterogeneity observed in the meta-analysis using Stata/SE 14.2, employing methods suggested by Miladinovic 2013. For health-related quality of life, a continuous outcome, we will use an alpha error as per guidance of Jakobsen 2014 (that is, 0.033 ), power of $90 \%$ (beta error of 10\%) (Castellini 2017), an SMD of 0.2, the median health-related quality of life in the control group in the trials, and the heterogeneity observed in the meta-analysis.

\section{Subgroup analysis and investigation of heterogeneity}

We plan to assess the differences in the effect estimates between the following subgroups and investigate heterogeneity and inconsistency using meta-regression with the help of the codes provided in NICE DSU guidance if we include a sufficient number of trials (at least two trials in at least two different categories) (Dias 2012a). We plan to use the following trial-level covariates for meta-regression.

- Trials at low risk of bias compared to trials at high risk of bias.

- Based on previous history of bleeding oesophageal varices.

- Based on the presence of other features of decompensation (for example, ascites).

- Based on the aetiology for cirrhosis (for example, alcoholrelated liver disease, viral liver diseases, autoimmune liver disease).

- Based on the severity of cirrhosis prior to the bleeding episode (for example, assessed by Child-Pugh score).

- Based on the interval between the variceal bleed and the start of treatment.

- Based on the cointerventions (for example, both groups receive prophylactic antibiotics to decrease the risk of subacute bacterial peritonitis in people with low-protein ascites).

- Based on the definition used by study authors for serious adverse events and any adverse events (ICH-GCP 1997 versus other definitions).

We will calculate a single common interaction term when applicable (Dias 2012a). If the $95 \% \mathrm{Crl}$ of the interaction term does not overlap zero, we will consider this statistically significant heterogeneity or inconsistency (depending upon the factor being used as covariate).

\section{Sensitivity analysis}

If a trial reports only per-protocol analysis results, we plan to reanalyse the results using the best-worst case scenario and worstbest case scenario analyses as sensitivity analyses whenever possible. We will also perform a sensitivity analysis excluding the trials in which the mean or standard deviation, or both were imputed, and we will use the median standard deviation in the trials to impute missing standard deviations.

We will compare our assessments of imprecision with GRADE methodology to that with Trial Sequential Analysis methodology (Castellini 2018).

\section{Presentation of results}

We will follow the PRISMA-NMA statement while reporting (Hutton 2015). We will present the effect estimates with 95\% CrI for each pair-wise comparison calculated from the direct comparisons and network meta-analysis. We will also present the cumulative probability of the treatment ranks (that is, the proba- 
bility that the intervention is within the top two, the probability that the intervention is within the top three, etc.) in graphs (SUCRA) (Salanti 2011). We will plot the probability that each intervention was best, second best, third best, etc. for each of the different outcomes (rankograms), which are generally considered more informative (Salanti 2011; Dias 2012b). We will also provide the $\mathrm{CrI}$ of the probabilities in the ranking probability tables. We will upload all the raw data and the codes used for analysis in The European Organization for Nuclear Research open source database (Zenodo) and provide a link within the review.

\section{Grading of evidence}

We will present 'Summary of findings' tables for all the primary and secondary outcomes (see Primary outcomes; Secondary outcomes). We will follow the approach suggested by Puhan and colleagues (Puhan 2014). First, we will calculate the direct and indirect effect estimates and 95\% CrI using the node-splitting approach (Dias 2010), that is, calculating the direct estimate for each comparison by including only trials in which there was direct comparison of interventions and the indirect estimate for each comparison by excluding the trials in which there was direct comparison of interventions. Next, we will rate the quality of direct and indirect effect estimates using GRADE methodology, which takes into account the risk of bias, inconsistency, directness of evidence, imprecision, and publication bias (Guyatt 2011). We will then present the estimates of the network meta-analysis and rate the quality of network meta-analysis effect estimates as the best quality of evidence between the direct and indirect estimates (Puhan 2014). In addition, we will present information on the absolute measures (that is, proportion of people with the outcome in each intervention group based on the direct estimates, indirect estimates, and network meta-analysis estimates). We will also present information on the number of trials and participants as per the standard 'Summary of findings' table.

\section{Recommendations for future research}

We will also provide recommendations for future research in the population, intervention, control, outcomes, period of follow-up, and study design based on the uncertainties that we identify from the existing research.

\section{ACKNOWLEDGEMENTS}

We acknowledge the help and support of the Cochrane HepatoBiliary Group. The authors would also like to thank the peer reviewers listed below who provided comments to improve the protocol.

Peer reviewers: Keith Lindor, USA; Beatriz Sanchez-Jimenez, Mexico.

Contact Editor: Christian Gluud, Denmark.

Sign-off Editor: Christian Gluud, Denmark.

Cochrane Review Group funding acknowledgement: The Danish State is the largest single funder of The Cochrane HepatoBiliary Group through its investment in The Copenhagen Trial Unit, Centre for Clinical Intervention Research, Rigshospitalet, Copenhagen University Hospital, Denmark.

This project was funded by the National Institute for Health Research (NIHR) Systematic Reviews Programme (project number $16 / 114 / 17)$.

\section{Department of Health Disclaimer}

The views and opinions expressed therein are those of the review authors and do not necessarily reflect those of the 16/114/17 Programme, NIHR, National Health Service, or the Department of Health.

\section{Danish State and The Copenhagen Trial Unit Disclaimer}

The views and opinions expressed in this protocol are those of the authors and do not necessarily reflect those of the Danish State or The Copenhagen Trial Unit. 


\section{REFERE N C E S}

\section{Additional references}

\section{Abby Philips 2016}

Abby Philips C, Sahney A. Oesophageal and gastric varices: historical aspects, classification and grading: everything in one place. Gastroenterology Report 2016;4(3):186-95.

\section{Adam 2012}

Adam R, Karam V, Delvart V, O'Grady J, Mirza D, Klempnauer J, et al. Evolution of indications and results of liver transplantation in Europe. A report from the European Liver Transplant Registry (ELTR). Journal of Hepatology 2012;57(3):675-88

\section{Beppu 1981}

Beppu K, Inokuchi K, Koyanagi N, Nakayama S, Sakata H, Kitano S, et al. Prediction of variceal hemorrhage by esophageal endoscopy. Gastrointestinal Endoscopy 1981;27 (4):213-8.

Best 2018

Best LM, Freeman S, Sutton AJ, Hawkins N, Tsochatzis E, Gurusamy KS. Treatment for hepatorenal syndrome in people with decompensated liver cirrhosis: a network metaanalysis. Cochrane Database of Systematic Reviews 2018, Issue 9. DOI: 10.1002/14651858.CD013103

\section{Cales 1990}

Cales P, Desmorat H, Vinel JP, Caucanas JP, Ravaud A, Gerin P, et al. Incidence of large oesophageal varices in patients with cirrhosis: application to prophylaxis of first bleeding. Gut 1990;31(11):1298-302.

\section{Castellini 2017}

Castellini G, Nielsen EE, Gluud C. Comment on: "Cell therapy for heart disease: trial sequential analyses of two Cochrane reviews". Clinical Pharmacology and Therapeutics 2017;102(1):21-4.

\section{Castellini 2018}

Castellini G, Bruschettini M, Gianola S, Gluud C, Moja L. Assessing imprecision in Cochranesystematic reviews: a comparison of GRADEand Trial Sequential Analysis. Systematic Reviews 2018;7(110):1-10.

\section{Chaimani 2012}

Chaimani A, Salanti G. Using network meta-analysis to evaluate the existence of small-study effects in a network of interventions. Research Synthesis Methods 2012;3(2): $161-76$.

\section{Chaimani 2013}

Chaimani A, Higgins JP, Mavridis D, Spyridonos P, Salanti G. Graphical tools for network meta-analysis in STATA. PloS One 2013;8(10):e76654.

\section{Chawla 2012}

Chawla S, Katz A, Attar BM, Gupta A, Sandhu DS, Agarwal R. Platelet count/spleen diameter ratio to predict the presence of esophageal varices in patients with cirrhosis: a systematic review. European Journal of Gastroenterology \& Hepatology 2012;24(4):431-6.
D’Amico 2010

D’Amico G, Pagliaro L, Pietrosi G, Tarantino I. Emergency sclerotherapy versus vasoactive drugs for bleeding oesophageal varices in cirrhotic patients. Cochrane Database of Systematic Reviews 2010, Issue 3. DOI: 10.1002/ 14651858.CD002233.pub2

\section{D'Amico 2014}

D’Amico G, Pasta L, Morabito A, D'Amico M, Caltagirone M, Malizia G, et al. Competing risks and prognostic stages of cirrhosis: a 25-year inception cohort study of 494 patients. Alimentary Pharmacology \& Therapeutics 2014;39 (10):1180-93.

\section{de Franchis 1992}

de Franchis R, Pascal JP, Ancona E, Burroughs AK, Henderson M, Fleig W, et al. Definitions, methodology and therapeutic strategies in portal hypertension. A consensus development workshop, Baveno, Lake Maggiore, Italy, April 5 and 6, 1990. Journal of Hepatology 1992;15(1-2):256-61.

\section{de Franchis 2015}

de Franchis R, Baveno VIF. Expanding consensus in portal hypertension: report of the Baveno VI consensus workshop: stratifying risk and individualizing care for portal hypertension. Journal of Hepatology 2015;63(3):743-52.

\section{Del Re 2013}

Del Re AC, Spielmans GI, Flückiger C, Wampold BE. Efficacy of new generation antidepressants: differences seem illusory. PloS One 2013;8(6):e63509.

\section{Dias 2010}

Dias S, Welton NJ, Caldwell DM, Ades AE. Checking consistency in mixed treatment comparison meta-analysis. Statistics in Medicine 2010;29(7-8):932-44.

\section{Dias 2012a}

Dias S, Sutton AJ, Welton NJ, Ades AE. NICE DSU technical support document 3: heterogeneity: subgroups, meta-regression, bias and bias-adjustment, September 2011 (last updated April 2012). nicedsu.org.uk/wpcontent/uploads/2016/03/TSD3-Heterogeneity.finalreport.08.05.12.pdf (accessed 17 July 2018).

\section{Dias 2012b}

Dias S, Welton NJ, Sutton AJ, Ades AE. NICE DSU technical support document 1: introduction to evidence synthesis for decision making, April 2011 (last updated April 2012). scharr.dept.shef.ac.uk/nicedsu/wp-content/uploads/ sites/7/2016/03/TSD1-Introduction.final_.08.05.12.pdf (accessed 17 July 2018)

\section{Dias 2014}

Dias S, Welton NJ, Sutton AJ, Caldwell DM, Lu G, Ades AE. NICE DSU technical support document 4: inconsistency in networks of evidence based on randomised controlled trials, May 2011 (last updated April 2014). nicedsu.org.uk/wp-content/uploads/2016/03/TSD4Inconsistency.final_.15April2014.pdf (accessed 17 July 2018). 


\section{Dias 2016}

Dias S, Welton NJ, Sutton AJ, Ades AE. NICE DSU technical support document 2: a generalised linear modelling framework for pairwise and network meta-analysis of randomised controlled trials, August 2011 (last updated September 2016). www.ncbi.nlm.nih.gov/pubmedhealth/ PMH0088912/pdf/PubMedHealth_PMH0088912.pdf (accessed 17 July 2018).

\section{EASL 2018}

European Association for the Study of the Liver. EASL Clinical Practice Guidelines for the management of patients with decompensated cirrhosis. Journal of Hepatology 2018; Vol. 69, issue 2:406-60. DOI: 10.1016/j.jhep.2018.03.024

\section{Ertel 2016}

Ertel AE, Chang AL, Kim Y, Shah SA. Management of gastrointestinal bleeding in patients with cirrhosis. Current Problems in Surgery 2016;53(8):366-95.

\section{EuroQol 2018}

EuroQol. EQ-5D Instruments | About EQ-5D, 2018. euroqol.org/eq-5d-instruments/ (accessed 17 July 2018).

\section{Fleming 2008}

Fleming KM, Aithal GP, Solaymani-Dodaran M, Card TR, West J. Incidence and prevalence of cirrhosis in the United Kingdom, 1992-2001: a general population-based study. Journal of Hepatology 2008;49(5):732-8.

\section{Garcia-Tsao 2017}

Garcia-Tsao G, Abraldes JG, Berzigotti A, Bosch J. Portal hypertensive bleeding in cirrhosis: risk stratification, diagnosis, and management: 2016 practice guidance by the American association for the study of liver diseases. Hepatology (Baltimore, Md.) 2017;65(1):310-35.

\section{Gines 2009}

Gines P, Schrier RW. Renal failure in cirrhosis. New England Journal of Medicine 2009;361(13):1279-90.

\section{Gluud 2012}

Gluud LL, Krag A. Banding ligation versus beta-blockers for primary prevention in oesophageal varices in adults. Cochrane Database of Systematic Reviews 2012, Issue 8. DOI: 10.1002/14651858.CD004544.pub2

\section{Gluud 2018}

Gluud C, Nikolova D, Klingenberg SL. Cochrane HepatoBiliary Group. About The Cochrane Collaboration (Cochrane Review Groups (CRGs)) 2018, Issue 3. Art. No.: LIVER.

\section{Gurusamy 2018}

Gurusamy K, Walmsley M, Davidson BR, Frier C, Fuller B, Madden A, et al. Top research priorities in liver and gallbladder disorders in the United Kingdom. Under review.

Guyatt 2011

Guyatt G, Oxman AD, Akl EA, Kunz R, Vist G, Brozek J, et al. GRADE guidelines: 1. Introduction - GRADE evidence profiles and summary of findings tables. Journal of Clinical Epidemiology 2011;64(4):383-94.

\section{Gøtzsche 2008}

Gøtzsche PC, Hrobjartsson A. Somatostatin analogues for acute bleeding oesophageal varices. Cochrane Database of Systematic Reviews 2008, Issue 3. DOI: 10.1002/ 14651858.CD000193.pub3

\section{Herman 2015}

Herman J, Baram M. Blood and volume resuscitation for variceal hemorrhage. Annals of the American Thoracic Society 2015;12(7):1100-2.

\section{Higgins 2011}

Higgins JP, Green S, editor(s). Cochrane Handbook for Systematic Reviews of Interventions Version 5.1.0 (updated March 2011). The Cochrane Collaboration, 2011. Available from handbook.cochrane.org.

\section{Higgins 2012}

Higgins JP, Jackson D, Barrett JK, Lu G, Ades AE, White IR. Consistency and inconsistency in network metaanalysis: concepts and models for multi-arm studies. Research Synthesis Methods 2012;3(2):98-110.

\section{Hutton 2015}

Hutton B, Salanti G, Caldwell DM, Chaimani A, Schmid $\mathrm{CH}$, Cameron $\mathrm{C}$, et al. The PRISMA extension statement for reporting of systematic reviews incorporating network meta-analyses of health care interventions: checklist and explanations. Annals of Internal Medicine 2015;162(11): 777-84.

\section{ICH-GCP 1997}

International Conference on Harmonisation Expert Working Group. International Conference on Harmonisation of Technical Requirements for Registration of Pharmaceuticals for Human Use. ICH Harmonised Tripartite Guideline. Guideline for Good Clinical Practice CFR \& ICH Guidelines. Vol. 1, Philadelphia (PA): Barnett International/PAREXEL, 1997.

Ioannou 2003 Ioannou GN, Doust J, Rockey DC. Terlipressin for acute esophageal variceal hemorrhage. Cochrane Database of Systematic Reviews 2003, Issue 1. DOI: 10.1002/ 14651858.CD002147

Jakobsen 2014 Jakobsen JC, Wetterslev J, Winkel P, Lange T, Gluud C. Thresholds for statistical and clinical significance in systematic reviews with meta-analytic methods. $B M C$ Medical Research Methodology 2014;14(1):120.

\section{Khan 2006}

Khan S, Tudur Smith C, Williamson P, Sutton R. Portosystemic shunts versus endoscopic therapy for variceal rebleeding in patients with cirrhosis. Cochrane Database of Systematic Reviews 2006, Issue 4. DOI: 10.1002/ 14651858.CD000553.pub2

\section{Kjaergard 2001}

Kjaergard LL, Villumsen J, Gluud C. Reported methodologic quality and discrepancies between large and small randomized trials in meta-analyses. Annals of Internal Medicine 2001;135(11):982-9. 


\section{Liu 2016}

Liu CL, Wu CK, Shi HY, Tai WC, Liang CM, Yang SC, et al. Medical expenses in treating acute esophageal variceal bleeding: a 15-year nationwide population-based cohort study. Medicine 2016;95(28):e4215.

\section{Lu 2006}

Lu G, Ades AE. Assessing evidence inconsistency in mixed treatment comparisons. Journal of the American Statistical Association 2006;101(474):447-59.

\section{Lundh 2017}

Lundh A, Sismondo S, Lexchin J, Busuioc OA, Bero L. Industry sponsorship and research outcome. Cochrane Database of Systematic Reviews 2017, Issue 2. DOI: 10.1002/14651858.MR000033.pub3

\section{McCarty 2017}

McCarty TR, Afinogenova Y, Njei B. Use of wireless capsule endoscopy for the diagnosis and grading of esophageal varices in patients with portal hypertension: a systematic review and meta-analysis. Journal of Clinical Gastroenterology 2017;51(2):174-82.

\section{McPherson 2016}

McPherson S, Lucey MR, Moriarty KJ. Decompensated alcohol related liver disease: acute management. BMJ (Clinical Research Ed.) 2016;352:1124.

\section{Merion 2010}

Merion RM. Current status and future of liver transplantation. Seminars in Liver Disease 2010;30(4): 411-21.

\section{Merli 2003}

Merli M, Nicolini G, Angeloni S, Rinaldi V, De Santis A, Merkel C, et al. Incidence and natural history of small esophageal varices in cirrhotic patients. Journal of Hepatology 2003;38(3):266-72.

\section{Miladinovic 2013}

Miladinovic J, Hozo I, Djulbegovic B. Trial sequential boundaries for cumulative meta-analyses. Stata Journal 2013;13(1):77-91.

\section{Mills 2012}

Mills EJ, Ioannidis JP, Thorlund K, Schünemann HJ, Puhan MA, Guyatt GH. How to use an article reporting a multiple treatment comparison meta-analysis. JAMA 2012; 308(12):1246-53.

\section{Moher 1998}

Moher D, Pham B, Jones A, Cook D J, Jadad AR, Moher $\mathrm{M}$, et al. Does quality of reports of randomised trials affect estimates of intervention efficacy reported in meta-analyses? Lancet 1998;352(9128):609-13.

\section{Mokdad 2014}

Mokdad AA, Lopez AD, Shahraz S, Lozano R, Mokdad AH, Stanaway J, et al. Liver cirrhosis mortality in 187 countries between 1980 and 2010: a systematic analysis. BMC Medicine 2014;12:145.
Moore 2013

Moore CM, Van Thiel DH. Cirrhotic ascites review: pathophysiology, diagnosis and management. World Journal of Hepatology 2013;5(5):251-63.

\section{NCBI 2018a}

National Center for Biotechnology Information. Liver cirrhosis. www.ncbi.nlm.nih.gov/mesh/68008103 (accessed on 17 July 2018).

\section{NCBI 2018b}

National Center for Biotechnology Information. Esophageal and gastric varices. www.ncbi.nlm.nih.gov/mesh/68004932 (accessed on 17 July 2018).

\section{Newell 1992}

Newell DJ. Intention-to-treat analysis: implications for quantitative and qualitative research. International Journal of Epidemiology 1992;21(5):837-41.

\section{NIEC 1988}

North Italian Endoscopic Club for the Study and Treatment of Esophageal Varices. Prediction of the first variceal hemorrhage in patients with cirrhosis of the liver and esophageal varices. New England Journal of Medicine 1988; 319(15):983-9.

\section{Olson 2016}

Olson JC, Saeian K. Gastrointestinal issues in liver disease. Critical Care Clinics 2016;32(3):371-84.

\section{Optum 2018}

Optum. Patient-reported Outcomes | What We Do | SF Health Surveys | SF-36v2 Health Survey, 2018. campaign.optum.com/optum-outcomes/what-we-do/ health-surveys/sf-36v2-health-survey.html (accessed on 14 April 2018).

\section{Perri 2016}

Perri GA, Khosravani H. Complications of end-stage liver disease. Canadian Family Physician Medecin de Famille Canadien 2016;62(1):44-50.

\section{Puhan 2014}

Puhan MA, Schünemann HJ, Murad MH, Li T, Brignardello-Petersen R, Singh JA, et al. A GRADE Working Group approach for rating the quality of treatment effect estimates from network meta-analysis. BMJ (Clinical Research Ed.) 2014;349:g5630.

Qi 2015

Qi XS, Bao YX, Bai M, Xu WD, Dai JN, Guo XZ. Nonselective beta-blockers in cirrhotic patients with no or small varices: a meta-analysis. World Journal of Gastroenterology 2015;21(10):3100-8.

\section{Ratib 2015}

Ratib S, Fleming KM, Crooks CJ, Walker AJ, West J. Causes of death in people with liver cirrhosis in England compared with the general population: a population-based cohort study. American Journal of Gastroenterology 2015; 110(8):1149-58.

\section{Read 1972}

Read AE. Clinical physiology of the liver. British Journal of Anaesthesia 1972;44(9):910-7. 
Rios 2015

Ríos CE, Seron P, Gisbert JP, Bonfill CX. Endoscopic injection of cyanoacrylate glue versus other endoscopic procedures for acute bleeding gastric varices in people with portal hypertension. Cochrane Database of Systematic Reviews 2015, Issue 5. DOI: 10.1002/14651858.CD010180.pub2

Royle 2003

Royle P, Milne R. Literature searching for randomized controlled trials used in Cochrane reviews: rapid versus exhaustive searches. International Journal of Technology Assessment in Health Care 2003;19(4):591-603.

\section{Salanti 2011}

Salanti G, Ades AE, Ioannidis JP. Graphical methods and numerical summaries for presenting results from multipletreatment meta-analysis: an overview and tutorial. Journal of Clinical Epidemiology 2011;64(2):163-71.

\section{Salanti 2012}

Salanti G. Indirect and mixed-treatment comparison, network, or multiple-treatments meta-analysis: many names, many benefits, many concerns for the next generation evidence synthesis tool. Research Synthesis Methods 2012;3(2):80-97.

Sass 2009

Sass DA, Chopra KB. Portal hypertension and variceal hemorrhage. Medical Clinics of North America 2009;93(4): 837-53, vii-viii.

\section{Savovic 2012a}

Savović J, Jones HE, Altman DG, Harris RJ, Jüni P, Pildal $\mathrm{J}$, et al. Influence of reported study design characteristics on intervention effect estimates from randomized controlled trials: combined analysis of meta-epidemiological studies. Health Technology Assessment 2012;16(35):1-82.

\section{Savović 2012b}

Savović J, Jones HE, Altman DG, Harris RJ, Jüni P, Pildal $\mathrm{J}$, et al. Influence of reported study design characteristics on intervention effect estimates from randomized controlled trials. Annals of Internal Medicine 2012;157(6):429-38.

Savovic 2018

Savović J, Turner RM, Mawdsley D, Jones HE, Beynon R, Higgins JP, et al. Association between risk-of-bias assessments and results of randomized trials in Cochrane Reviews: the ROBES Meta-Epidemiologic Study. American Journal of Epidemiology 2018;187(5):1113-22.

\section{Scaglione 2015}

Scaglione S, Kliethermes S, Cao G, Shoham D, Durazo $\mathrm{R}$, Luke A, et al. The epidemiology of cirrhosis in the United States: a population-based study. Journal of Clinical Gastroenterology 2015;49(8):690-6.

\section{Schulz 1995}

Schulz KF, Chalmers I, Hayes RJ, Altman DG. Empirical evidence of bias. Dimensions of methodological quality associated with estimates of treatment effects in controlled trials. JAMA 1995;273(5):408-12.

\section{Setiawan 2016}

Setiawan VW, Stram DO, Porcel J, Lu SC, Le Marchand L, Noureddin M. Prevalence of chronic liver disease and cirrhosis by underlying cause in understudied ethnic groups: the multiethnic cohort. Hepatology (Baltimore, Md.) 2016; 64(6):1969-77.

\section{Severini 1993}

Severini TA. Bayesian interval estimates which are also confidence intervals. Journal of the Royal Statistical Society. Series B, Statistical Methodology 1993;55(2):533-40.

\section{Thabut 2007}

Thabut D, Hammer M, Cai Y, Carbonell N. Cost of treatment of oesophageal variceal bleeding in patients with cirrhosis in France: results of a French survey. European Journal of Gastroenterology and Hepatology 2007;19(8): 679-86.

\section{Thorlund 2011}

Thorlund K, Engstrøm J, Wetterslev J, Brok J, Imberger G, Gluud C. User manual for Trial Sequential Analysis (TSA), 2011. ctu.dk/tsa/files/tsa_manual.pdf (accessed 17 July 2018).

\section{Thorlund 2012}

Thorlund K, Mills EJ. Sample size and power considerations in network meta-analysis. Systematic Reviews 2012;1:41.

\section{Tripathi 2015}

Tripathi D, Stanley AJ, Hayes PC, Patch D, Millson C, Mehrzad H, et al. U.K. Guidelines on the management of variceal haemorrhage in cirrhotic patients. Gut 2015;64 (11):1680-704.

\section{TSA 2011 [Computer program]} Copenhagen Trial Unit. TSA - Trial Sequential Analysis. Version 0.9.5.10 Beta. Copenhagen: Copenhagen Trial Unit, 2011.

\section{Tsochatzis 2014}

Tsochatzis EA, Bosch J, Burroughs AK. Liver cirrhosis. Lancet 2014;383(9930):1749-61.

\section{Turner 2012}

Turner RM, Davey J, Clarke MJ, Thompson SG, Higgins JP. Predicting the extent of heterogeneity in meta-analysis, using empirical data from the Cochrane Database of Systematic Reviews. International Journal of Epidemiology 2012;41(3):818-27.

van Valkenhoef 2012

van Valkenhoef G, Lu G, de Brock B, Hillege H, Ades AE, Welton NJ. Automating network meta-analysis. Research Synthesis Methods 2012;3(4):285-99.

Villanueva 2013

Villanueva C, Colomo A, Bosch A, Concepcion M, Hernandez-Gea V, Aracil C, et al. Transfusion strategies for acute upper gastrointestinal bleeding. New England Journal of Medicine 2013;368(1):11-21.

\section{Wetterslev 2008}

Wetterslev J, Thorlund K, Brok J, Gluud C. Trial sequential analysis may establish when firm evidence is reached in 
cumulative meta-analysis. Journal of Clinical Epidemiology 2008;61(1):64-75.

\section{Wetterslev 2017}

Wetterslev J, Jakobsen JC, Gluud C. Trial Sequential Analysis in systematic reviews with meta-analysis. BMC Medical Research Methodology 2017;17(1):39.

\section{Williams 2014}

Williams R, Aspinall R, Bellis M, Camps-Walsh G, Cramp $\mathrm{M}$, Dhawan A, et al. Addressing liver disease in the UK: a blueprint for attaining excellence in health care and reducing premature mortality from lifestyle issues of excess consumption of alcohol, obesity, and viral hepatitis. Lancet
2014;384(9958):1953-97.

\section{Wood 2008}

Wood L, Egger M, Gluud LL, Schulz KF, Juni P, Altman DG, et al. Empirical evidence of bias in treatment effect estimates in controlled trials with different interventions and outcomes: meta-epidemiological study. BMJ (Clinical Research Ed.) 2008;336(7644):601-5.

\section{Zaman 2000}

Zaman A, Goldberg RJ, Pettit KG, Kaniecki DJ, Benner $\mathrm{K}$, Zacker C, et al. Cost of treating an episode of variceal bleeding in a VA setting. American Journal of Gastroenterology 2000;95(5):1323-30.

* Indicates the major publication for the study

\section{A P P E N D I C E S}

\section{Appendix I. Search strategies}

\begin{tabular}{|c|c|c|}
\hline Database & Time span & Search strategy \\
\hline $\begin{array}{l}\text { Central Register of Controlled Trials } \\
\text { (CENTRAL) in the Cochrane Library }\end{array}$ & Latest issue & $\begin{array}{l}\# 1 \mathrm{MeSH} \text { descriptor: [Esophageal and Gas- } \\
\text { tric Varices] explode all trees } \\
\# 2 * \text { esophageal varic }{ }^{*} \\
\# 3 \# 1 \text { or } \# 2\end{array}$ \\
\hline MEDLINE Ovid & January 1947 to date of search & $\begin{array}{l}\text { 1. exp "Esophageal and Gastric Varices"/ } \\
\text { 2. *esophageal varic*/.ti,ab. } \\
\text { 3. } 1 \text { or } 2 \\
\text { 4. randomized controlled trial.pt. } \\
\text { 5. controlled clinical trial.pt. } \\
\text { 6. randomized.ab. } \\
\text { 7. placebo.ab. } \\
\text { 8. drug therapy.fs. } \\
\text { 9. randomly.ab. } \\
\text { 10. trial.ab. } \\
\text { 11. groups.ab. } \\
\text { 12. } 4 \text { or } 5 \text { or } 6 \text { or } 7 \text { or } 8 \text { or } 9 \text { or } 10 \text { or } 11 \\
\text { 13. exp animals/ not humans.sh. } \\
\text { 14. } 12 \text { not } 13 \\
\text { 15. } 3 \text { and } 14\end{array}$ \\
\hline Embase Ovid & January 1974 to date of search & $\begin{array}{l}\text { 1. exp esophagus varices/ } \\
\text { 2. *esophageal varic*/.ti,ab. } \\
\text { 3. } 1 \text { or } 2 \\
\text { 4. exp crossover-procedure/ or exp double- }\end{array}$ \\
\hline
\end{tabular}

Treatment for bleeding oesophageal varices in people with decompensated liver cirrhosis: a network meta-analysis (Protocol) 


\begin{tabular}{|c|c|c|}
\hline & & $\begin{array}{l}\text { blind procedure/ or exp randomized con- } \\
\text { trolled trial/ or single-blind procedure/ } \\
5 .(((((\text { random* or factorial* or crossover* } \\
\text { or cross over* or cross-over* or placebo* or } \\
\left.\left.\left.\left.\text { double }^{*}\right) \text { adj blind }{ }^{*}\right) \text { or single* }\right) \text { adj blind* }\right) \\
\text { or assign* or allocat* or volunteer* }) . a f \\
6.4 \text { or } 5 \\
7.3 \text { and } 6\end{array}$ \\
\hline $\begin{array}{l}\text { Science Citation Index Expanded (Web of } \\
\text { Science) }\end{array}$ & January 1945 to date of search & $\begin{array}{l}\text { \#1 TS }=\left({ }^{*} \text { esophageal varic* }\right) \\
\# 2 \mathrm{TS}=\left(\text { random* OR } \text { rct* OR crossover }^{*} \text { OR }\right. \\
\text { OR masked OR blind* OR placebo* OR } \\
\text { meta-analysis OR systematic review* OR } \\
\text { meta-analys })\end{array}$ \\
\hline $\begin{array}{l}\text { World Health Organization International } \\
\text { Clinical Trials Registry Platform ( } \\
\text { apps.who.int/trialsearch/Default.aspx) }\end{array}$ & $\begin{array}{l}\text { Date of search to be provided at the review } \\
\text { stage }\end{array}$ & Condition: Esophageal Varices \\
\hline ClinicalTrials.gov & $\begin{array}{l}\text { Date of search to be provided at the review } \\
\text { stage }\end{array}$ & Interventional Studies | Esophageal Varices \\
\hline $\begin{array}{l}\text { European Medical Agency } \\
\text { (www.ema.europa.eu/ema/) and US Food } \\
\text { and Drug Administration (www.fda.gov) }\end{array}$ & $\begin{array}{l}\text { Date of search to be provided at the review } \\
\text { stage }\end{array}$ & Esophageal Varices; random \\
\hline
\end{tabular}

\section{Appendix 2. Sample size calculation}

The short-term mortality of an episode of acute variceal bleeding is about $15 \%$ to $30 \%$ (Ioannou 2003; Gøtzsche 2008; D'Amico 2010; Rios 2015). The required information size based on a control group proportion of $20 \%$, a relative risk reduction of $20 \%$ in the experimental group, type I error of 5\%, and type II error of 20\% is 1447 participants. Network analyses are more prone to risk of random errors than direct comparisons (Del Re 2013). Accordingly, a greater sample size is required in indirect comparisons than in direct comparisons (Thorlund 2012). The power and precision in indirect comparisons depend upon various factors, such as the number of participants included for each comparison and the heterogeneity between the trials (Thorlund 2012). If there is no heterogeneity across the trials, the sample size in indirect comparisons would be equivalent to the sample size in direct comparisons. The effective indirect sample size can be calculated using the number of participants included in each direct comparison (Thorlund 2012). For example, a sample size of 2500 participants in the direct comparison A versus $C\left(\mathrm{n}_{A C}\right)$ and a sample size of 7500 participants in the direct comparison B versus $C\left(n_{B C}\right)$ results in an effective indirect sample size of 1876 participants. However, in the presence of heterogeneity within the comparisons, the required sample size is higher. In the above scenario, for an $\mathrm{I}^{2}$ statistic for each of the comparisons $\mathrm{A}$ versus $\mathrm{C}\left(\mathrm{I}_{A C}{ }^{2}\right)$ and $\mathrm{B}$ versus $\mathrm{C}\left(\mathrm{I}_{B C}{ }^{2}\right)$ of $25 \%$, the effective indirect sample size is 1407 participants. For an $\mathrm{I}^{2}$ statistic for each of the comparisons A versus $\mathrm{C}$ and $\mathrm{B}$ versus $\mathrm{C}$ of $50 \%$, the effective indirect sample size is 938 participants (Thorlund 2012). If there are only three groups and the sample size in the trials is more than the required information size, we will calculate the effective indirect sample size using the following generic formula (Thorlund 2012):

$\left(\mathrm{n}_{A C} \times\left(1-\mathrm{I}_{A C}{ }^{2}\right)\right) \times\left(\mathrm{n}_{B C} \times\left(1-\mathrm{I}_{B C}{ }^{2}\right)\right) /\left(\mathrm{n}_{A C} \times\left(1-\mathrm{I}_{A C}{ }^{2}\right)\right)+\left(\mathrm{n}_{B C} \times\left(1-\mathrm{I}_{B C}{ }^{2}\right)\right)$.

Currently, there is currently no method to calculate the effective indirect sample size for a network analysis involving more than three intervention groups.

Treatment for bleeding oesophageal varices in people with decompensated liver cirrhosis: a network meta-analysis (Protocol) 


\section{CONTRIBUTIONSOFAUTHORS}

Conceiving the protocol: KG, DR, ET.

Designing the protocol: KG, DR

Co-ordinating the protocol: KG.

Designing search strategies: KG.

Writing the protocol: DR, KG.

Providing general advice on the protocol: ET.

Securing funding for the protocol: KG.

All authors approved of the current protocol version.

Performing previous work that was the foundation of the current study: not applicable.

\section{DECLARATIONS OF INTEREST}

DR: none known.

ET: none known.

KG: none known.

\section{SOURCES OF SUPPORT}

\section{Internal sources}

- University College London, UK.

Writing equipment, software etc

\section{External sources}

- National Institute for Health Research, UK.

Payment for writing reviews, writing equipment, software

\section{NOTES}

The methods section of this protocol is based on a standard template used by Cochrane Hepato-Biliary Group modified for network meta-analysis used by the author group based on advice provided by the Complex Reviews Support Unit for a network meta-analysis protocol (Best 2018).

Treatment for bleeding oesophageal varices in people with decompensated liver cirrhosis: a network meta-analysis (Protocol) 